\title{
Instruments and techniques for the analysis of wheelchair propulsion and upper extremity involvement in patients with spinal cord injuries: current concept review
}

\author{
Fabio Dellabiancia1 \\ Giuseppe Porcellini² \\ Giovanni Merolla ${ }^{1,2}$
}

1 Biomechanics Laboratory "Marco Simoncelli", D.
Cervesi Hospital, Cattolica, Rimini, Italy
2 Unit of Shoulder and Elbow Surgery, D. Cervesi
Hospital, Cattolica, Rimini, Italy

Correspondig author:

Giovanni Merolla

Biomechanics Laboratory "Marco Simoncelli"

Unit of Shoulder and Elbow Surgery

D. Cervesi Hospital

Via L. V. Beethoven 1,

47841 Cattolica, Rimini, Italy

E-mail: giovannimerolla@hotmail.com, giovanni.merolla@auslrn.net

\section{Summary}

The correct functionality of the upper limbs is an essential condition for the autonomy of people with disabilities, especially for those in wheelchair. In this review we focused on the biomechanics of wheelchair propulsion and we described the instrumental analysis of techniques for the acquisition of wheelchair propulsion.

KEY WORDS: shoulder, wheelchair, propulsion, cord injury.

\section{Introduction}

The correct functionality of the upper limbs is an essential condition for the autonomy of people with disabilities, especially for those in wheelchair ${ }^{1}$.

The preservation of anatomy and physiology of the shoulder is the most critical factor, because this joint is at a high risk of deterioration, particularly for individuals in wheelchair ${ }^{2}$. A deepened understanding of the mechanisms and causes that lead to such impairment is performed by specific instruments, with a significant impact on the daily life of these patients. An efficient quantitative assessment of the upper limb's condition assists the clinician in the development of an adequate prevention and intervention plan.

The analysis of kinematics and dynamics of wheelchair motion is useful to define the indices that quan- tify the degree of inefficiency during specific tests or daily actions.

Aim of this review was to describe on the instrumental analysis of techniques for the acquisition of wheelchair propulsion.

The use of these methodologies is still controversial due to inconsistency in the performance of tests and consequent results reported in the literature ${ }^{3}$.

In order to obtain quantitative parameters, we focus on the biomechanics of wheelchair propulsion using electromyographic, kinematic and dynamic techniques.

\section{Biomechanics and kinematics of wheelchair propulsion}

Wheelchair propulsion is a repetitive task which is primarily controlled by the upper extremity and leads to biomechanical alterations that play a key role in triggering muscle imbalance and pain.

We distinguish two phases in the propulsion: pushing and recovery ${ }^{4-6}$ (Fig. 1A, B). During the first phase the hands partially follow the circular motion and the wheel can be moved imparting a tangential force upward with the elbow flexed (between the beginning and the middle of the push) and one downward with the elbow extended (from the middle to the end of the movement) ${ }^{7}$ (Fig. 2). Although the greatest loads act in the shoulder articulation, a further contribution of push is exerted by the wrist ${ }^{4}$. As results, the gradual change of the tangential force applied to the wheel has a detrimental effect on rotator cuff ${ }^{4}$. The rotator cuff is composed by 4 muscles (supraspinatus, infraspinatus, teres minor, subscapularis) and its actions are specific to the angular positions of the arm and the type of activity that is performed. Supraspinatus is an initiator of abduction and lies in the scapular plane, infraspinatus and teres minor are external rotators of the shoulder and lie below the scapular spine while subscapularis is the main internal rotator of the shoulder, the largest and strongest ${ }^{8}$.

In a normal shoulder the deltoid is the most powerful muscle and can generate a force up to 6 times the weight of the arm. It has the largest moment arm around the glenohumeral joint and is the most effective muscle in arm elevation, acting superiorly at $63^{\circ}$ when the arm is resting at the side of the body. The deltoid can alone provide $70 \%$ of the torque at $30^{\circ}$ of abduction and $85 \%$ at $90^{\circ}$, the supraspinatus acts 15 $20^{\circ}$ superiorly and can generate a force of 2.5 times the weight of the arm, the infraspinatus acts $40-45^{\circ}$ inferiorly and the force of the teres minor is also directed inferiorly at $55^{\circ}$. Together, the infraspinatus 
Instruments and techniques for the analysis of wheelchair propulsion and upper extremity involvement in patients with spinal cord injuries: current concept review

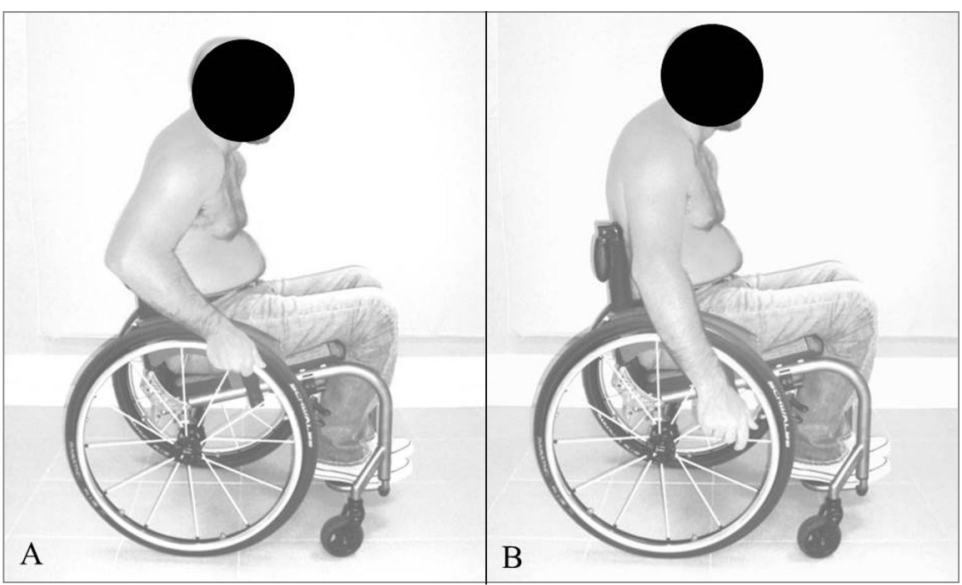

Figure 1. Wheelchair propulsion: push (A) and recovery $(\mathrm{B})$ phase.

Figure 2. Trajectory of the hands during the two phases of propulsion. S: shoulder, E: elbow, 1: start push, 2 end push, 3 start recovery, 4 end recovery.
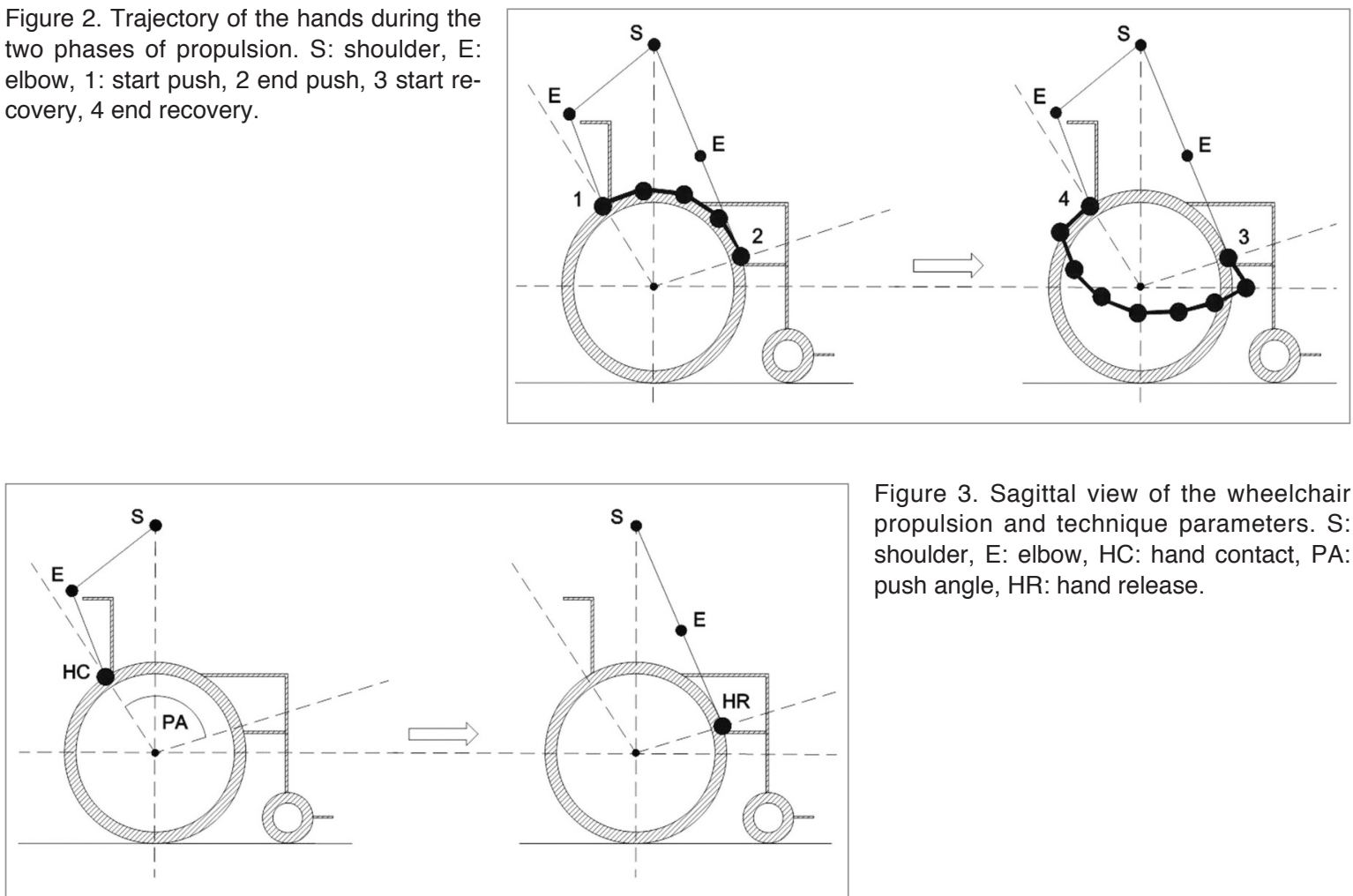

Figure 3. Sagittal view of the wheelchair propulsion and technique parameters. S: shoulder, E: elbow, HC: hand contact, PA: push angle, HR: hand release.

and teres minor can generate a force of 5 times the weight of the $\mathrm{arm}^{9}$.

When abduction of the arm is initiated, the shear force on the glenohumeral joint generated by the deltoid is counteracted by the compressive force produced by the rotator cuff. Generally, the compressive force contributes towards joint stability while the shear force induces upward displacement of the humeral head. The combination of shear and compressive forces allows efficient abduction by stabilising the humeral head within the glenoid fossa. The magnitude and direction of the resultant joint reaction force is dependent on the position of the arm. At $90^{\circ}$ of abduction, the joint reaction force is at its maximum and equates approximately the weight of the body ${ }^{9}$.
During propulsion, the shoulder is maintained at approximately $70^{\circ}$ of abduction. At the onset of the propulsive phase of motion, the shoulder is extended and internally rotated and subsequently ends up flexed and externally rotated at the onset of the recovery phase (Fig. 3). As a result of years of wheelchair propulsion, wheelchairs users often have well-developed shoulder flexors, internal rotators, and adductors, but may have poorly developed external rotators and thoracoscapular muscles ${ }^{10}$. This muscular imbalance and the repetitive nature of the wheelchair push predispose to the subacromial impingement ${ }^{4}$.

The elbow is flexed throughout the pushing phase, starting from an angle of about $60^{\circ}$, gradually increasing until the hand assume the most distant position 
from the ground and subsequently decreasing until to reach the minimum distance from the ground near by the recovery phase ${ }^{11}$ (Fig. 3).

Anterior deltoid, pectoralis major and biceps brachii act primarily during the pushing phase and contraction start towards the end of the recovery phase with load peaks at around $10 \%$ of the pushing phase; conversely, the activity of the triceps brachii is initially quite modest during this phase, and then it gradually increases until the time of the release of the hand ${ }^{5}$. The deactivation occurs in the final pushing phase, where the muscles of the recovery phase begin to act: middle and posterior deltoid, subscapularis, supraspinatus and medium trapezius ${ }^{4,5}$.

\section{Wheelchair features}

Patients with spinal cord injuries use a wheelchair that could be moved manually pushing the wheels. The features required in a wheelchair depend upon user characteristics and intended activities. The components include wheels, tyres, castors, frames, materials, construction details, seats, backrests, brakes, handrims, footrests and armrests. Each component is considered in relation to performance characteristics including rolling resistance, versatility, weight, comfort, stability, transfer, durability and maintenance ${ }^{12-14}$. The pulling force varied with the weight and the rolling resistance of different tyres. For example a high pressure pneumatic tyre required only one quarter of the pulling force of the solid grey rubber tyres (which were in common use throughout the United States). Synthetic tyre can be designed to be much more durable, cheaper, lighter and with a rolling resistance comparable to pneumatics. The diameter of the tyres also has a significant effect. As a general rule, the rolling resistance is inversely proportional to the diameter ${ }^{12-14}$.

The material from which a handrim is made is an important factor. Plywood has a pleasant feel and appearance, but is much too expensive. Generally the rim is made of metal to dissipate heat while braking. Metal handrims may be aluminium, chrome plated steel, or stainless steel. Vinyl and other plastics are used as a coating over metal rims to increase friction. Softer foam covers have also been introduced, to increase gripping friction and to avoid injury to insensitive hands ${ }^{12-14}$.

Wheelchairs use castors to move in any direction. The basic castor consists of a wheel, an axle, a fork and a stem. Wheels are available in several sizes. A smaller wheel has greater probability to damage than larger. Damage occurs from impact with obstacles such as kerbs and pot-holes ${ }^{12-14}$.

Although some generalization can be made regarding the materials and construction of the frame, the overall design should depend upon the characteristics of the user. For example a simple lightweight frame may be ideal for an athletic active user ${ }^{12-14}$.

Adjustments and material properties of the seat and backrest have been much studied over the years.
The common material for both has been reinforced vinyl fabric. It is moisture proof, abrasion resistant and easily cleaned but also exhibits undesirable properties such as stretching. Common adjustments are seatback angle and seatback height ${ }^{12-14}$.

Footrest and armrest may be subject to high load during inadverted impact or lifting. Footrests are adjustable and easily removed for transfer in and out of the wheelchair while armrests are fixed and thus may interfere with transfer ${ }^{12-14}$

Manual wheelchairs are equipped with brakes to stop or while descending a slope (accomplished by friction to the handrim). Some wheelchairs are equipped with dynamic brakes that can be used for both functions. These are of special value to those with impaired hand function or where hills are frequently encountered $^{12-14}$.

\section{Instruments and techniques for analysis of propulsion}

The techniques adopted for the instrumental evaluation of the wheelchair propulsion involve the use of electromyography, stereophotogrammetric and sensoristic systems and Smart Wheel to analyze the dynamics of the motion $6,15-18$. In addition, specific 3-D models investigate wheelchair propulsion and biomechanical parameters that vary during joint motion (angles, speeds, accelerations, joint forces and moments).

Several studies focused on electromyographic analysis of muscle fatigue. Most ${ }^{6,18-21}$ focused on no more than 5 muscles (anterior and posterior deltoid, pectoralis major, biceps brachii and triceps brachii), others analyzed $7^{22,23}$ (anterior, medium and posterior deltoid, pectoralis major, medium trapezius, biceps brachii, triceps brachii) or 12 muscles ${ }^{5,24-26}$ (anterior, medium and posterior deltoid, pectoralis major, latissimus dorsi, serratus, trapezius, supraspinatus, infraspinatus, subscapularis, biceps brachii and triceps brachii). Compared to the common representation of the two phases of propulsion ${ }^{4-6}$, Louis and Gorce 23 introduce two additional sub-steps in the pushing phase (initial and final for a total of three phases).

It is necessary to refer to specific standards to place electrodes on muscles. Kloosterman et al. ${ }^{22}$ and Louis and Gorce ${ }^{23}$ used the SENIAM (Surface Electromyography Non-Invasive Assessment of Muscle), Europeans recommendations for placement on 27 muscles site across the following areas: shoulder and neck, the back muscles, the arm and hand, the upper leg and hip and the lower leg27.

Additional studies reported a detailed description of the aforementioned tests, in particular Dubowsky et al. ${ }^{19}$ referred to Anatomical Guide For The Electromyographer 28 , that information for approximately 93 appendicular and 22 axial muscles including innervation and attachments of each muscle, how to position the patient for the examination, the appropriate site for insertion of the electrode and the action that the patient should perform to activate the muscle 28 . 
Instruments and techniques for the analysis of wheelchair propulsion and upper extremity involvement in patients with spinal cord injuries: current concept review

Lin et al. ${ }^{18}$ used the protocol by Chopp et al. ${ }^{29}$, that electrodes over the infraspinatus, sternal pectoralis major, and latissimus dorsi. The diameter of the electrodes used to record data ranges from $20 \mathrm{~mm}^{18}$ to $40 \mathrm{~mm}^{23}$.

Most of studies specified that the electromyograph was bipolar5, 6, 18-22 and used adhesive tape to fix the electrodes on the skin and to fasten together the elettromyographic wires, in order to limit the effect of interference due to sliding of the electrodes on the skin (motion artifact of soft tissues). Alternatively modern wireless electromyograph have been described ${ }^{22}$.

The electromyographic data were recorded at variable frequencies and filtered with Butterworth filters of different orders also with variable recording times. Data are sampled at a frequency of $50 \mathrm{~Hz}$ and filtered with Butterworth filter of the second order with cutoff frequency of $10 \mathrm{~Hz}^{5}, 20,24$.

Kinematic and dynamic techniques have been widely described. Specifically have been analysed kinematic using stereophotogrammetric systems ${ }^{16}, 17,19,20$, 22-26, 30-33, electromagnetic sensors ${ }^{15}, 30,3-\mathrm{D}$ biomechanical models $6,16,17,22,24-26,32,34,35$ and SmartWheel to record the dynamic of propulsion 15-17, 19, 22, 24-26, 31, 34, 36 . The stereophotogrammetry uses a minimum of three cameras $^{26,34}$ up to a maximum of ten ${ }^{32}$ to monitor the position of articular segments placing special reflective anatomical markers.

Since there is no agreement regards the number and the positioning of markers, several authors refer to the International Society of Biomechanics (ISB) ${ }^{37}$.

The markers are positioned on the chest, upper arm, forearm and hand and on the respective joints of the shoulder, elbow and wrist, unilaterally 16, 17, 19, 20, 22-24, $26,32-34$ or bilaterally 25,31 , furthermore the same aforementioned authors also applied the markers on the wheelchair to define the orientation in the global coordinate system.

Arnet et al. ${ }^{24}$ and Morrow et al. ${ }^{32}$ use cluster of markers composed respectively by three and four markers each. Morrow et al. ${ }^{32}$ applies a single cluster of markers near the sternum; Arnet et al. ${ }^{24}$ applies five of these on trunk, acromion, arm, forearm and hand.

The kinematic data are acquired at variable frequencies, in a range from $50^{17}$ to $240 \mathrm{~Hz}^{25}$; data are subsequently filtered with Butterworth filters of variable order, zero phase, with frequency ranging from 6 to $40 \mathrm{~Hz}$.

Raina et al. ${ }^{15}$ and Riek et al. ${ }^{30}$ propose a kinematic analysis using electromagnetic or inertial sensors and these are applied directly on the body of the subject. The systems used are respectively "PCIBird" and "Flock of Birds": the first uses five sensors, interfaced with the software "Motion Monitor", placed on T1 vertebra, acromion, humerus, near the scapula and the brachioradialis muscle of the forearm, the second records chest, scapula and humerus orientation with a fewer number of sensors.

Necessary to the study of the kinematics is accurate using 3-D dynamic models to define the body segments. Although biomechanical musculoskeletal models differ in the number and in the structure of articu- lar segments considered the trunk and the humerus are included in overall models ${ }^{6,25,26,32}$. Each joint is commonly represented by six degrees of freedom ${ }^{26}$ except for Louis and Gorce ${ }^{23}$ who represented the joints with only three degrees of freedom.

The models are obtained from specific software 6,16 , 17, 22, 24, 26, 32, 34. The models most used are the Delft Shoulder and Elbow Model ${ }^{38}$ and the Dutch Shoulder Model $^{39}$ that use rigid bodies and actuators to simulate muscle action. All the models refer to Euler angles to describe the position of the various integral segments with the rigid body (through a rotations series starting from a fixed coordinate reference system).

The most commonly procedure used for the analysis of articular forces and moments involves the use of SmartWheel ${ }^{16,17, ~ 19, ~ 22, ~ 24-26, ~ 31, ~ 36 . ~ K w a r c i a k ~ e t ~ a l . ~}{ }^{36}$ and Rankin et al. ${ }^{26}$ also specified the model "OptiPush". Raina et al. ${ }^{15}$ and Van Drongelen et al. ${ }^{34}$ instead exploited particular sensors positioned near the wheelchair. Masse et al. ${ }^{20}$ analyzes these variables (joint forces and moments) using the software "Biomech".

Such systems sample data at a variable frequencies, from $100^{24}$ to $240 \mathrm{~Hz}^{25}$. Butterworth filters of variables orders (II, IV, VIII), low-pass, cut-off $20 \mathrm{~Hz}$ are used to filter the data. The dynamic data are resampled and synchronized with the kinematic data at frequencies from 50 to $120 \mathrm{~Hz}^{16,24,25,31}$.

With regard to data processing, these are directly transferred from the workstation used for the acquisition to the software MatLab ${ }^{15}, 16,19,22,23,25,32,36$ or data can be transferred first to Microsoft Excel and subsequently to MatLab ${ }^{19,25}$.

\section{Discussion}

About $70 \%$ of disabled people using wheelchairs complains of pain and injuries from wear and tear in the upper limbs ${ }^{40}$, essentially due to high loads that occur during pushing phase and to the low mechanical efficiency of the device ${ }^{41}$, related to the difficulties of the patients to impart a perfectly tangential force to the wheel ${ }^{42}$. Some authors ${ }^{43,44}$ showed that an efficient push is mechanically not effective, in fact the complex set of adjustments that the have to face, makes the movement physiologically advantageous but biomechanically imperfect ${ }^{45,46}$.

The main pathologies that result from this excess load include subacromial impingement, long head of the biceps tendonitis, glenohumeral osteoarthritis and rotator cuff injuries $8,18,32$.

Wheelchair propulsion can be interpreted by specific dynamic models represented by biomechanical variables (angles, speed and angular accelerations, positioning of the marker, electromyographic data, etc.) that vary during the execution of the movement, consequently it is mandatory to define the correct coordinate system ${ }^{33}$. In particular during a repetitive motion, biomechanical variables show a cyclic behavior $^{4}$. This overuse causes muscle fatigue in the upper limb and this is the main cause of injury for paraplegics population ${ }^{16}$. 
Muscle fatigue is monitored by elettromyograph and is defined as a change of the signal following a sustained contraction in time that precedes the inability to protract the effort required ${ }^{4,47,48}$.

Electromyographic findings of the wheelchair propulsion are controversial ${ }^{5,6,18}$. The most fatigued muscle is the supraspinatus, that appears to be the only active muscle in both phases of the propulsion ${ }^{5}$. Rankin et al. ${ }^{6}$ showed in a study on twenty subjects, that the middle deltoid is the most fatigued one, followed by pectoralis major, anterior deltoid and infraspinatus and in the same order of activation they contribute in power during the push phase. In the recovery phase, the posterior deltoid produced considerably more force than all other muscles ${ }^{5}$. The peak of muscular activity of the biceps brachii was found at hand contact, while triceps brachii increased progressively during the push phase, reaching maximal values at hand release ${ }^{49}$.

Several research compared the muscle activity of the subjects with spinal cord injury to non-disabled subjects showing different muscle activation and timing of the pushing phase ${ }^{19,22,23}$.

Arnet et al. ${ }^{24}$ and Kloosterman et al. ${ }^{22}$, monitoring muscle activity during propulsion on both power-assisted wheelchair and hand-bike agreed that glenohumeral contact forces and relative muscle forces decrease and they concluded that thus may help prevent overuse injuries of the shoulder complex.

With regard to the ergonomics of the wheelchair, Masse et al. ${ }^{20}$ and Yang et al. ${ }^{31}$ agreed that the seat tilted backwards confers greater stability and increasing the seat height, the activities of biceps brachii and pectoralis major decrease.

It is therefore essential to evaluate kinematics and dynamics of the scapulothoracic and glenohumeral joints during propulsion. Despite wheelchair propulsion has been studied for about forty years, there is still no validated protocol for kinematic and dynamic analysis of this motor gesture ${ }^{3}$.

Stereophotogrammetric and sensoristic analysis are the most used instruments in kinematic analysis.

Collinger et al. ${ }^{16}$ showed significant correlations between kinematics-height and kinematics-weight at different velocity. These variables were forced into a regression models, particularly height was only a significant predictor of shoulder extension moment while weight seemed to be the primary factor contributing to shoulder kinetics. At all speed conditions, increased subject weight was predictive of higher anterior, posterior, inferior and lateral forces.

Newsam et al. ${ }^{11}$ and Lin et al. ${ }^{18}$ found that movement patterns vary depending on wheelchair configuration, level of injury and speed.

Sensoristic analysis uses portable electromagnetic sensors to describe movements of joints and segments ${ }^{15}, 30$. Sensors, that are less bulky and more simple in preparation, have as main limitation magnetic construction components that can produce interference near metal parts ${ }^{15,30}$.

Electromagnetic and inertial sensors are mainly exploited for the analysis of scapular orientation, essen- tial together with humerus to determine acromial space. In particular, Raina et al. ${ }^{15}$ and Riek et al. ${ }^{30}$ reported similar results with regard to the movement of scapular tilt (rotation anterior/posterior of the scapula, lateral view) and rotation of the glenohumeral joint. Morrow et al. ${ }^{32}$ showed that the scapula remained internally rotated during level and ramp propulsion. The scapula also exhibited slight upward rotation throughout the activity for all conditions. During level and ramp propulsion, peak superior intersegmental loading occurred immediately before the end of the push phase prior to the hand leaving the rim $^{35}$.

The definition and the choose of a coordinate reference system is a complex and laborious process that begin defining segments from basic physiological movements and placing on each segment a coordinate system. Special 3-D models are exploited to establish which body segments consider, that differ by number of segments and joints ${ }^{25}$.

The technology most exploited in order to study the dynamics of propulsion involves the use of SmartWheel that is particular wheelchair equipped with a mechanical device for data recording 4,50 . Since this device is generally placed on only one side of the wheelchair may induce slight imbalance that affects the propulsion phases.

Desroches et al. ${ }^{33}$ showed that the highest moment found at the wrist joint was in extension and peak extension moment occurred at $20 \%$ of the propulsive cycle. The 3-D angle between the wrist joint moment and joint angular velocity vectors showed that the joint is in a stabilization configuration when the hand contacts the pushrim. The highest joint moment found at the elbow was in flexion and peak flexion moments occurred at $10 \%$ of the propulsive cycle. According to the 3-D angle, the elbow joint is in a propulsion configuration in the first $10 \%$ of the propulsive cycle, from $11 \%$ to the end of the propulsive cycle, the elbow joint was found to be in a stabilization configuration. Flexion, internal rotation and abduction were respectively the highest moments found at the shoulder joint. Peak flexion moment was revealed at $19 \%$ of the propulsive cycle, peak internal rotation moment at $21 \%$ and peak abduction at $48 \%$. During the entire push phase, the 3-D angle revealed mainly a propulsion configuration for the shoulder joint. Near the end of the push phase $(40 \%$ propulsive cycle), the joint shifts from a propulsion to a stabilization configuration.

Finally all studies use special wheelchairs designed to be applied on a treadmill ${ }^{36}$. All handrim biomechanics (contact angle, peak force, average force, and peak axle moment) were found to be similar and highly correlated. Based on the results by Kwarciak et al. ${ }^{36}$, a motor-driven treadmill can serve as a valid surrogate for overground studies of wheelchair propulsion.

In conclusion, the analysis of the current literature showed that the combined and contemporary use of the entire instrumentation group is the best strategy, providing a great amount of data to achieve an easier and accurate analysis of wheelchair propulsion that hel- 
Instruments and techniques for the analysis of wheelchair propulsion and upper extremity involvement in patients with spinal cord injuries: current concept review

ps to understand the physiopathology of articular and musculotendinous injuries in paraplegic population.

\section{References}

1. Shimada SD, Robertson RN, Bonninger ML, Cooper RA. Kinematic characterization of wheelchair propulsion. J Rehabil Res Dev 1998; 35:210-218.

2. Jain NB, Higgins LD, Katz JN, Garshick E. Association of shoulder pain with the use of mobility device in person with chronic spinal cord injury. PM R 2010; 2:896-900.

3. Lentino C, Checchia GA, Massone A, Sanguineti V, Basteris A, Vigo G. Analisi cinematica della spinta in carrozzina: proposta di modello sperimentale. Eur Med Phys 2008; 44:1-3.

4. Qi L. Use of wavelet analysis techniques with surface EMG and MMG to characterise motor uit recruitment patterns of shoulder muscles during wheelchair propulsion and voluntary contraction tasks. PhD thesis, Institute of Orthopaedics and Musculoskeletal Science; University College London.

5. Mulroy SJ, Gronley JK, Newsam CJ, Perry J. Electromyographic activity of shoulder muscles during wheelchair propulsion by paraplegic persons. Arch Phys Med Rehabil 1996; 77:187-193.

6. Rankin JW, Richter WM, Neptune RR. Individual muscle contributions to push and recovery subtasks during wheelchair propulsion. J Biomech 2011; 44:1246-1252.

7. Guo LY, Zhao KD, Su FC, An KN. Moment generation in wheelchair propulsion. Proc Inst Mech Eng H 2003; 217:405-413

8. Villaseñor-Ovies $\mathrm{P}$, Vargas A, Chiapas-Gasca K, Canoso JJ, Hernández-Díaz C, Saavedra MÁ, Navarro-Zarza JE, Kalish RA. Clinical anatomy of the elbow and shoulder. Reumatol Clin 2012; 8:13-24.

9. Lam F, Bhatia DN, Mostof SB, van Rooyen K, de Beer JF. Biomechanical considerations of the normal and rotator cuff deficient shoulders and the reverse shoulder prosthesis. Curr Orthopaed 2007; 21:40-46.

10. Ambrosio F, Boninger ML, Souza AL, Fitzgerald SG, Koontz AM, Cooper RA. Biomechanics and strength of manual wheelchair users. J Spinal Cord Med 2005; 28:407-414.

11. Newsam CJ, Rao SS, Mulroy SJ, Gronley, JK, Bontrager EL, Perry J. Three dimensional upper extremity motion during manual wheelchair propulsion in men with different levels of spinal cord injury. Gait Posture 1999; 10:223-232.

12. McLaurin CA, Brubaker CE. Biomechanics and the wheelchair. Prosthet Orthot Int 1991; 15:24-37.

13. Liu HY, Pearlman J, Cooper R, Hong EK, Wang H, Salatin $\mathrm{B}$, Cooper RA. Evaluation of aluminum ultralight rigid wheelchairs versus other ultralight wheelchairs using ANSI/RESNA standards. J Rehabil Res Dev 2010; 47:441-456.

14. Pazzaglia M, Galli G, Scivoletto G, Molinari M. A functionally relevant tool for the body following spinal cord injury. PLoS One 2013; 8:e58312.

15. Raina S, McNitt-Gray JL, Mulroy S, Requejo PS. Effect of increased load on scapular kinematics during manual wheelchair propulsion in individuals with paraplegia and tetraplegia. Hum Movement Sci 2012; 31:397-407.

16. Collinger JL, Boninger ML, Koontz AM, Price R, Sisto SA, Tolerico ML, Cooper RA. Shoulder biomechanics during the push phase of wheelchair propulsion: a multisite study of persons with paraplegia. Arch Phys Med Rehabil 2008; 89:667-676.

17. Gil-Agudo A, Del Ama-Espinosa A, Perez-Rizo E, PerezNombela S, Pablo Rodrıguez-Rodrıguez L. Upper limb joint kinetics during manual wheelchair propulsion in patients with different levels of spinal cord injury. J Biomech 2010; 43:2508-2515.

18. Koontz AM, Lin YS, Worobey L, Boninger ML. Effect of muscle fatiguing tasks on subacromial space in manual wheelchair users. Rehabilitation Engeneering and Assistive Technology Society of North America 2012 Annual Conference, June 28-July 3, 2012, Baltimore.

19. Dubowsky SR, Sisto SA, Langrana NA. Comparison of kinematics, kinetics, and emg throughout wheelchair propulsion in able-bodied and persons with paraplegia: An Integrative Approach. J Biomech Eng 2009; 131:021015.

20. Masse LC, Lamontagne M, O'Riain MD. Biomechanical analysis of wheelchair propulsion for various seating positions. $J$ Rehabil Res Dev 1992; 29:12-28.

21. Bernasconi SM, Tordi N, Ruiz J, Parratte B. Changes in oxigen uptake, shoulder muscle activity and propulsion cycle timing during strenuos wheelchair exercise. Spinal cord 2007; 45:468-474.

22. Kloosterman MG, Eising $\mathrm{H}$, Schaake L, Buurke JH, Rietman JS. Comparison of shoulder load during power assisted and purely hand-rim wheelchair propulsion. Clin Biomech 2012; 27:428-435.

23. Louis N, Gorce P. Surface electromiography activity of upper limb muscle during wheelchair propulsion: influence of wheelchair configuration. Clin Biomech 2010; 25:879-885.

24. Arnet U, van Drongelen S, Scheel-Sailer A, van der Woude LHV, Veeger DH. Shoulder load during synchronous handcycling and handrim wheelchair propulsion in persons with paraplegia. J Rehabil Med 2012; 44:222-228.

25. Dubowsky SR, Rasmussen J, Sisto SA, Langrana NA. Validation of a musculoskeletal model of wheelchair propulsion and its application to minimizing shoulder joint forces. J Biomech 2008; 41:2981-2988.

26. Rankin JW, Kwarciak AM, Richter WM, Neptune RR. The influence of wheelchair propulsion technique on upper extremity muscle demand: a simulation study. Clin Biomech 2012; 27:879-886.

27. Hermens JH, Freriks B, Disselhorst-Klug C, Rau G. Development of recommendations for SEMG sensors and sensor placement procedures. J Electromyogr Kines 2000; 10:361-374

28. Perotto AO, Delagi EF, Lazetti J, Morison D. Anatomical Guide For The Electromyographer: the limbs and the trunks. Charles C Thomas Publisher 2005; 10:345.

29. Chopp JN, Fischer SL, Dickerson CR. The specificity of fatiguing protocols affects scapular orientation: implications for subacromial impingement. Clin Biomech 2011; 26:40-45.

30. Riek LM, Ludewig PM, Nawoczenski DA. Comparative shoulder kinematics during free standing, standing depression lifts and daily functional activities in persons with paraplegia: considerations for shoulder health. Spinal Cord 2008; 46:335-343.

31. Yang YS, Koontz AM, Yeh SJ, Chang JJ. Effect of backrest height on wheelchair propulsion biomechanics for level and uphill conditions. Arch Phys Med Rehabil 2012; 93:654-659.

32. Morrow MM, Kaufman KR, An KN. Scapula kinematics and associated impingement risk in manual wheelchair users during propulsion and a weight relief lift. Clin Biomech 2011; 26:352-357.

33. Desroches G, Dumas R, Pradon D, Vaslin P, Lepoutre FX, Chèze L. Upper limb joint dynamics during manual wheelchair propulsion. Clin Biomech 2010; 25:299-306.

34. van Drongelen S, van der Woude LHV, Veeger HEJ. Load on the shoulder complex during wheelchair propulsion and weight relief lifting. Clin Biomech 2011; 26:452-457.

35. Morrow MM, Hurd WJ, Kaufman KR, An KN. Upper extremity joint kinetics expression during wheelchair propulsion. J Rehabil Res Dev 2009; 46:939-944. 
36. Kwarciak AM, Turner JT, Guo L, Richter WM,. Comparing handrim biomechanics for treadmill and overground wheelchair propulsion. Spinal Cord 2010; 49:457-462.

37. Wu G, van der Helm FCT, Veeger HEJ, Makhsous M, Van Roy P, Anglin C, Nagels J, Karduna AR, McQuade K, Wangk $X$, Werner FW, Buchholz B. ISB recommendation on definitions of joint coordinate systems of various joints for the reporting of human joint motion - Part II: shoulder, elbow, wrist and hand. J Biomech 2005; 38:981-992.

38. Van der Helm FC. Analysis of the kinematic and dynamic behavior of the shoulder mechanism. J Biomech 1994; 27:527-550.

39. Van der Helm FC, Veeger HE, Pronk GM, van der Woude LH, Rozendal RH. Geometry parameters for musculoskeletal modelling of the shoulder system. J Biomech 1992; 25:129-144.

40. Finley MA, Rodgers MM. Prevalence and identification of shoulder pathology in athletic and nonathletic wheelchair users with shoulder pain: A pilot study. J Rehabil Res Dev 2004; 41:395-402.

41. Mercer JL, Boninger M, Koontz A, Ren D, Dyson-Hudson T, Cooper R. shoulder joints kinetics and pathology in manual wheelchair users. Clin Biomech 2006; 21:781-789.

42. Boninger ML, Cooper RA, Robertson RN, Shimada SD. Three-dimensional pushrim forces during two speeds of wheelchair propulsion. Am J Phys Med Rehab 1997; 76:420-426.
43. De Groot S, Veeger HEJ, Hollander AP, van der Woude LHV. Consequence of feedback-based learning of an effective hand rim wheelchair force production on mechanical efficiency. Clin Biomech 2002; 17:219-226.

44. De Groot S, Veeger HEJ, Hollander AP, van der Woude LHV. Effect of wheelchair stroke pattern on mechanical efficiency. Am J Phys Med Rehab 2004; 83:640-649.

45. Bregman DJ, van Drongelen S, Veeger HE. Is effective force application in handrim wheelchair propulsion also efficient? Clin Biomech 2009; 24:13-19.

46. Dallmejer AJ, van der Woude LHV, Veeger HEJ, Hollander AP. Effectiveness of force application in manual wheelchair propulsion in persons with spinal cord injuries. Am J Phys Med Rehab 1998; 77:213-221.

47. Conforto S, Mathieu P, Schmid M, Bibbo D, Florestal JR, D'Alessio T. How much can we trust the electromechanical delay estimated by using electromyography? Conf Proc IEEE Eng Med Biol Soc 2006; 1:1256-1259.

48. Roberts TJ, Gabaldón AM. Interpreting muscle function from EMG: lessons learned from direct measurements of muscle force. Integr Comp Biol 2008; 48:312-320.

49. Chow JW, Millikan TA, Carlton LG, Morse MI, Chae WS. Biomechanical comparison of two racing wheelchair propulsion techniques. Med Sci Sport Exer 2001; 33:476-484.

50. Cooper RA. SmartWheel: from concept to clinical practice. Prosthet Orthot Int 2009; 33:198-209. 